\title{
Vascular Sciatica
}

\section{Tino Emanuele Poloni ${ }^{1 *}$, Alberto Galli ${ }^{2}$, Eugenio Benericetti ${ }^{3}$, Mauro Ceroni ${ }^{4}$}

${ }^{1}$ Service of Neurology; Fondazione Cenci-Golgi/ASP-Golgi Redaelli, via. San Martino n. 10-20081 Abbiategrasso, Milan, Italy

${ }^{2}$ UO of Neurology, AO Ospedale San Carlo Borromeo, Milan, Italy

${ }^{3}$ UO Neurosurgery, Maria Cecilia Hospital Private Hospital; Cotignola, Ravenna, Italy

"IRCCS Foundation "National Neurological Institute C. Mondino", Department of Neurological Sciences, University of Pavia, Pavia, Italy

We present the operating photograph of a young men who presented a chronic distressful right sciatic pain exacerbated by internal rotation of the extended leg and by abduction and external rotation of the thigh (Pace's sign, elicited by the contraction of piriformis muscle). The patient had no neurological deficit. Magnetic Resonance Imaging of the spine, lumbosacral plexus, and sciatic nerve was normal. This clinical picture was suggestive of a piriformis muscle syndrome [1].

Surgical exploration of the greater ischial foramen, through the buttock, disclosed a bipartition of the right sciatic nerve (S). The sciatic nerve was divided by an arterial loop (A) which derived from collateral of the inferior gluteal artery. Just below the inferior margin of the piriformis muscle $(\mathrm{P})$, a fibrous coating wrapped the vascular anomaly and the nerve. The arterial pulse was transmitted to the nerve. The cautious lysis of the fibrous coating and the interposition of a Gore-tex patch between the nerve and the anomalous vessel eliminated the vascular conflict. After the operation the pain was no longer perceptible. Unfortunately, several years later the pain gradually recurred especially during physical activity. A second operation

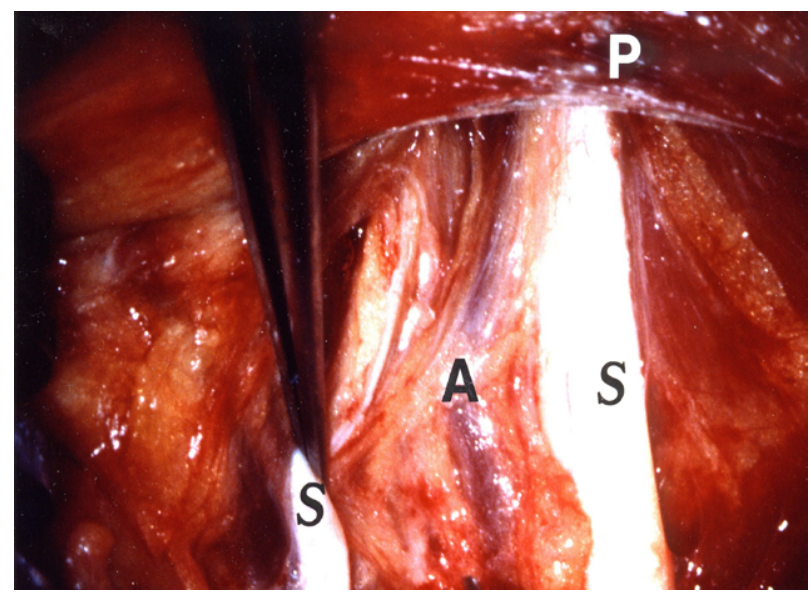

revealed a chronic inflammatory reaction, probably due to the friction of the Gore-tex patch against the nerve. A careful removal of the patch and inflammatory tissue allowed a significant pain relief.

Vascular malformations are a very rare cause of sciatic pain of extraspinal origin and few cases are reported also in recent series [2]. To our best knowledge, this picture represents a unique anomaly characterized by a bipartition of sciatic nerve associated with anomalous collateral of the inferior gluteal artery. Moreover we note that Gore-tex patches are excellent for neuro-vascular conflicts involving nerves in static conditions, such as cranial nerves, but their use must be cautious in nerves subject to movement.

\section{References}

1. Beatty RA (1994) The piriformis muscle syndrome: a simple diagnostic maneuver. Neurosurgery 34: 512-514.

2. Van Gompel JJ, Griessenauer CJ, Scheithauer BW, Amrami KK, Spinner RJ (2010) Vascular malformations, rare causes of sciatic neuropathy: a case series. Neurosurgery 67: 1133-1142.
*Corresponding author: Tino Emanuele Poloni, Service of Neurology Fondazione Cenci Golgi /ASP-Golgi Redaelli, Milan, Italy,Tel: +39029466409; Fax: +390294608148; E-mail: tepoloni@libero.it, e.poloni@golgicenci.it

Received August 22, 2014; Accepted August 27, 2014; Published August 30 2014

Citation: Poloni TE, Galli A, Benericetti E, Ceroni M (2014) Vascular Sciatica. J Clin Case Rep 4: I101. doi:10.4172/2165-7920.1000I101

Copyright: $\odot 2014$ Poloni TE, et al. This is an open-access article distributed under the terms of the Creative Commons Attribution License, which permits unrestricted use, distribution, and reproduction in any medium, provided the original author and source are credited. 